\title{
Correction: Confirmatory multiparametric magnetic resonance imaging at recruitment confers prolonged stay in active surveillance and decreases the rate of upgrading at follow-up
}

\author{
Stefano Luzzago - Michele Catellani - Ettore Di Trapani - Gabriele Cozzi (i) - Francesco A. Mistretta • \\ Roberto Bianchi · Paola Pricolo - Andrea Conti - Eleonora Ancona - Nicolò Piacentini - Sarah Alessi · \\ Giuseppe Renne - Matteo Ferro - Deliu-Victor Matei - Gennaro Musi - Barbara Alicja Jereczek-Fossa • \\ Giuseppe Petralia $\cdot$ Ottavio de Cobelli
}

Published online: 13 December 2019

(c) The Author(s), under exclusive licence to Springer Nature Limited 2019

\section{Correction to: Prostate Cancer and Prostatic Diseases https://doi.org/10.1038/s41391-019-0160-3}

Since publication of the original paper, the authors realised that the following incorrect denomination of the IEO Institute was used in several affiliations: "Istituto Europeo di
Oncologia (IEO)". According to the recent rules of the Italian Ministry of Health the correct denomination is "IEO, European Institute of Oncology IRCCS, Milan". This has been corrected in the appropriate affiliations in both the HTML and PDF versions of this paper. 